\title{
Gastric Schwannoma
}

Tanawat Pattarapuntakul, M.D.

Nanthana-Kriangkrai Chotiwattanaphan Institute of Gastroenterology and Hepatology, Faculty of Medicine, Prince of Songkla University, Hat Yai, Songkhla 90110, Thailand.

Received 30 August 2017 • Accepted 26 January 2018 • Published online 24 May 2018

\section{Abstract:}

A gastric schwannoma is a rare primary spindle cell mesenchymal tumor of the digestive tract. Gastric schwannomas originate from Schwann cells of the peripheral nerves in the stomach. The majority of schwannomas are benign, slow growing tumors only a few of which develop into malignancies. Due to their indolent course, in most cases, gastric schwannomas are asymptomatic or discovered as an incidental finding on cross-sectional imaging or endoscopy. When symptomatic, the most common presenting symptoms are abdominal pain, upper gastrointestinal bleeding and intraabdominal mass. Preoperatively, gastric schwannomas are difficult to differentiate from other mesenchymal tumors, such as gastrointestinal stroma or leiomyoma which develop from mesenchymal stem cells. The optimal management of the tumor is based on the symptoms of the patient, tumor size and histologic grading and the prognosis is excellent after complete surgical or endoscopic removal. Gastric schwannomas need multidisciplinary team management for definitive diagnosis and management, including specialists from gastroenterology, surgery, radiology and pathology.

Keywords: gastric schwannoma, gastric subepithelial tumors 


\section{Introduction}

A schwannoma is a rare primary spindle cell mesenchymal tumor of the digestive tract, about $73.0 \%$ of which are found in the stomach. Gastric schwannomas originate from Schwann cells of the peripheral nerves in the stomach, and represent about $2.0-7.0 \%$ of benign gastric tumors. ${ }^{1,2}$ The majority of schwannomas are benign, slow growing tumors, only a few of which develop into malignancies. ${ }^{3}$ There is a female predilection, with peak incidence in the 5th and 6th decades of life. The gastric schwannoma was first reported by Daimaru et al., in 1988, with confirmed diagnosis by histology and immunohistochemistry (positive S100 and glial fibrillary acidic protein). ${ }^{4}$ Due to their indolent course, in most cases, gastric schwannomas are asymptomatic or discovered as an incidental finding on crosssectional imaging or endoscopy. When symptomatic, the common presenting symptoms are abdominal pain, upper gastrointestinal bleeding and intra-abdominal mass. Preoperatively, gastric schwannomas are difficult to differentiate from other mesenchymal tumors, such as gastrointestinal stromal tumors (interstitial cells of Cajai) or leiomyoma (smooth muscle) which develop from mesenchymal stem cells. ${ }^{5}$ Gastric schwannomas need multidisciplinary team management for definitive diagnosis and management, including specialist from gastroenterology, surgery, radiology and pathology.

\section{Pathogenesis}

The gastric schwannoma arises from the sheath of Auerbach's plexus or, less commonly, from Meissner's plexus. The tumors are generally benign, often asymptomatic, and usually discovered incidentally from cross-sectional imaging, endoscopy, or intra-operatively. The tumor shares the characteristics of an exophytic mass extending from the gastric wall to the abdominal cavity, which have similar histology to other gastric subepithelial tumors such as gastrointestinal stromal tumors, leiomyomas and leiomyosarcomas., These tumors are mostly single lesion, vary in size, and are composed of spindle cell forming sheets, which have a high blood supply but intra-tumoral slow blood flow causing overlying mucosal ischemia causing mucosal ulceration. ${ }^{2}$

Previous studies have noted a genetic association of the gastric schwannoma to monosomy variants of chromosome 22 and somatic NF2 gene mutations. These variants are more commonly associated with soft tissue schwannomas than gastric schwannomas, however these genetic variants can be found in gastric schwannomas with associated NF2 gene mutation in the presence of multiple tumor lesions and other organ involvement. ${ }^{7-11}$

\section{Prevalence and clinical manifestations}

The gastric schwannoma is a primary benign mesenchymal gastric tumor, with slow growth and low malignancy potential. The incidence rate is about $0.2-1.0 \%$ of all gastric tumors and $2.0-7.0 \%$ of benign gastric mesenchymal tumors, occurring about 2-4 times more frequently in females during the $5^{\text {th }}$ and $6^{\text {th }}$ decades. Due to their indolent course, in most cases, gastric schwannomas are asymptomatic or discovered as an incidental finding on crosssectional imaging or endoscopy. The common presenting symptoms are abdominal pain and upper gastrointestinal (GI) bleeding, weight loss and gastric outlet obstruction (Table 1). ${ }^{6,8}$ The gastric schwannoma varies in size from 1 to $10.5 \mathrm{~cm}$ in diameter and can be found in any part of the stomach wall that has a peripheral nerve sheath structure (Table 2). ${ }^{6}$

Table 1 Clinical manifestations of gastric schwannoma $(n=80)^{6,8}$

\begin{tabular}{ll}
\hline Clinical manifestations & Number (\%) \\
\hline Asymptomatic & $25(31.3)$ \\
Abdominal pain & $22(27.5)$ \\
Upper Gl bleeding & $10(6.1)$ \\
Anorexia and weight loss & $2(2.5)$ \\
Gastric outlet obstruction & $1(1.3)$ \\
No data & $18(22.5)$ \\
\hline
\end{tabular}

Gl=gastrointestinal 
Table 2 The common locations of gastric schwannoma $(n=29)^{6}$

\begin{tabular}{ll}
\hline Location & Number (\%) \\
\hline Gastric antrum & $10(34.5)$ \\
Greater curvature & $7(24.1)$ \\
Gastric fundus & $6(20.7)$ \\
Lesser curvature & $5(17.2)$ \\
Gastric cardia & $1(3.4)$ \\
\hline
\end{tabular}

\section{Diagnosis}

The gastric schwannoma is a rare primary mesenchymal tumor of the stomach which needs a multidisciplinary approach for diagnosis and management, involving specialists in gastroenterology, surgery, radiology and pathology.

\section{Radiography}

The gastric schwannoma is one of the primary gastric mesenchymal tumors, composed of spindle cells and with a high blood supply, but with an intra-tumoral slow blood flow causing overlying mucosal ischemia and subsequent mucosal ulceration. The computerized tomography findings of this tumor are shown in Table $3 .^{12}$

A computerized tomography (CT) scan of a gastric schwannoma usually shows a single lesion with a round or oval appearance, a thin wall capsule, and exophytic growth pattern. Hemodynamically the tumor has a homogenous enhancement pattern in the arterial phase without cystic or hemorrhagic components (Figure 1). An magnetic resonance imaging (MRI) shows a low signal intensity on T1-weighted images and high signal intensity on T2-weighted images, without cystic or hemorrhagic components, and high intensity on diffusion weighted imaging. ${ }^{12-14}$ The finding of adjacent significant intra-abdominal lymph nodes, recurrent tumor or evidence of metastasis to other organs is considered to be an indication of a malignant schwannoma.
Table 3 Computerized tomography (CT) scan findings in gastric schwannoma $(n=16)^{12}$

\begin{tabular}{ll}
\hline CT scan & Number (\%) \\
\hline Contour & $12(75.0)$ \\
$\quad$ Round or oval & $4(25.0)$ \\
Lobulate & \\
Margin & $16(100.0)$ \\
$\quad$ Discrete & \\
Growth pattern & $1(6.3)$ \\
$\quad$ Endoluminal & $9(56.3)$ \\
Exogastric & $6(37.5)$ \\
Both & $3(18.8)$ \\
Ulceration & \\
Cystic changes & $2(12.5)$ \\
Present & $14(87.5)$ \\
Absent & \\
Enhancement pattern & $13(81.3)$ \\
Homogenous & $3(18.8)$ \\
Heterogenous &
\end{tabular}

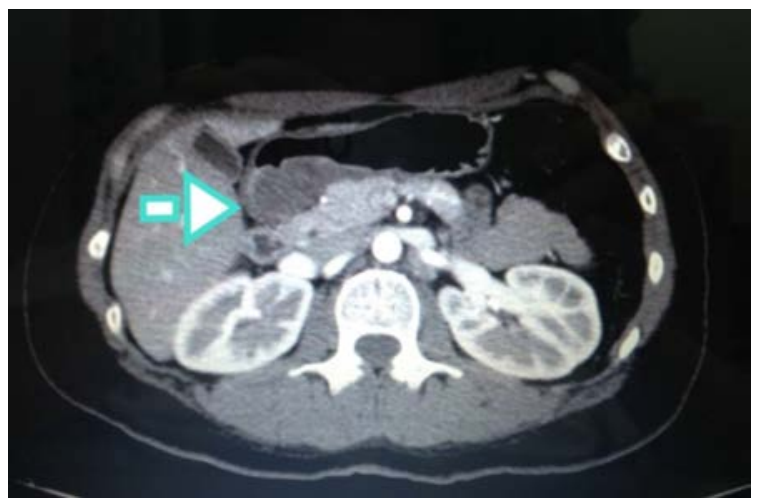

Figure 1 A CT scan of the abdomen showing a well demarcated, round, $2^{*} 3.5^{\star} 5.4 \mathrm{~cm}$, heterogenous, contrastenhanced mass with ulceration at the gastric antrum without cystic of hemorrhagic components 


\section{Endoscopy and endoscopic ultrasound}

The standard upper endoscopic examination of a schwannoma shows a subepithelial mass with or without ulcerations on top of the tumor, but it should be noted that this finding is not specific to a schwannoma and can be found in other subepithelial tumors (Figure 2). An endoscopic ultrasound can improve the diagnosis of tumors in the originative layer of the tumor and intra-tumoral components, which can lead to a more accurate diagnosis of subepithelial tumors (Figure 3 and Table 4).
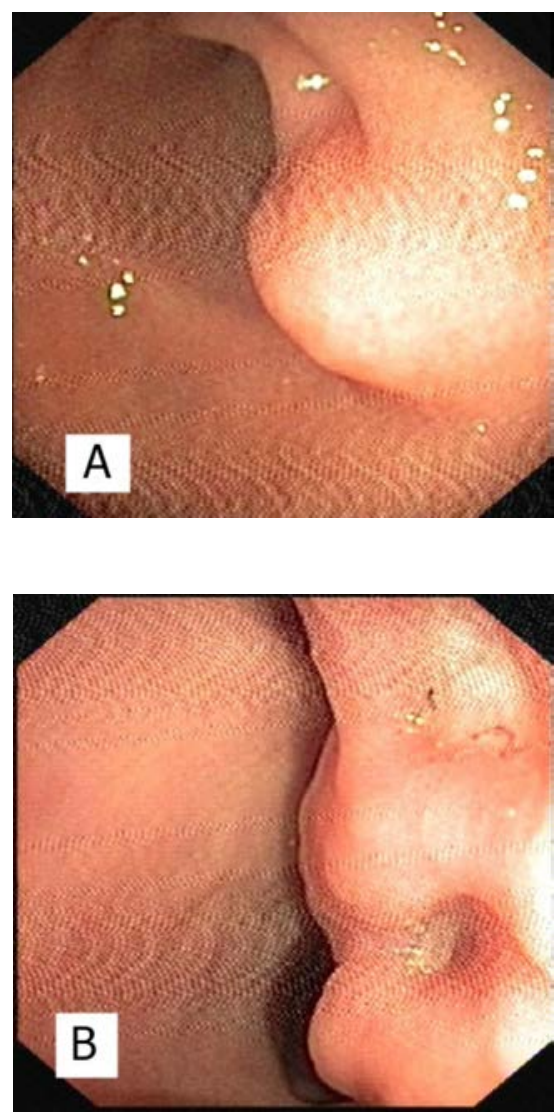

Figure 2 Endoscopic white-light showing a lobulated contour subepithelial mass $3^{*} 3 \mathrm{~cm}$ in diameter $(\mathrm{A})$ with a pigmented ulcer on a mucosal tumor at the gastric antrum (B).

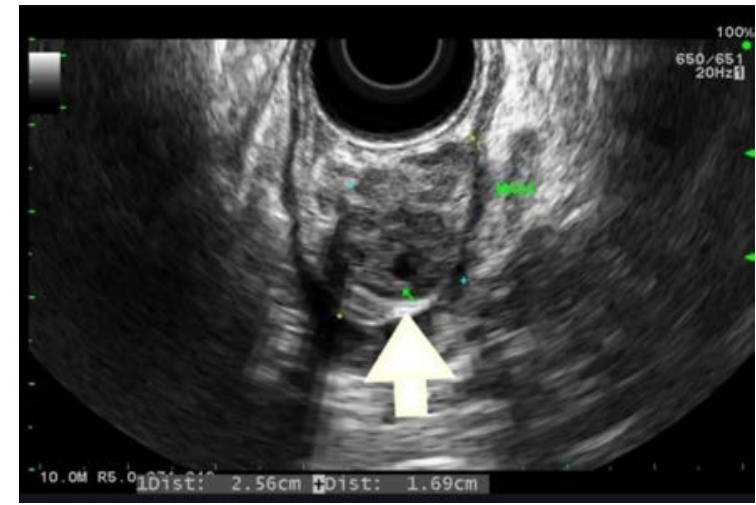

Figure 3 An endoscopic ultrasound showing a subepithelial heterogeneous hypoechoic mass size $2.68 * 1.67$ $\mathrm{cm}$ that originated from the third layer of the stomach wall with a hypoechoic tubular structure seen in the central mass and cystic degeneration or ductal structure.

Previous studies have noted some different endoscopic ultrasound (EUS) findings which can help to distinguish schwannomas from gastrointestinal stromal tumors (GISTs). The EUS findings in a schwannoma are a heterogenous and hypoechoic pattern which is higher than the muscularis propria and with an internal high echo area (indicating slow intratumoral blood flow) and these tumors less commonly featurecystic degeneration, hemorrhage or calcification (Table 5). ${ }^{16}$ However, although the EUS may be useful, appropriate histology is the gold standard for definitive diagnosis or EUS, with fine needle aspiration (FNA) also of benefit. Mekky et al. reported in 2010 that, EUS in combination with FNA in gastric subepithelial tumors got an appropriate tissue about $83.0 \%$ with a high rate of definite diagnosis of $96.3 \%$. $^{1,18}$

\section{Pathology}

Gastric schwannomas do not have specific biochemical assay or tumor markers which allow noninvasive testing, and need histology and immunohisto- 
chemistry for definitive diagnosis. The gross pathology finding of this tumor is a round thin wall capsular mass, whitish to pale or yellowish with a shiny color and whorled appearance without cystic degeneration or hemorrhage in the tumor. ${ }^{6,8}$

Microscopic findings show a spindle shaped cell with nuclear palisading and no definite true capsules with microscopic ulceration present in $\mathbf{8 3 . 0 \%}$. Ninety-six percent of the tumors show lymphocytic cuffing at the periphera part and about $61.0 \%$ have diffuse intratumoral lymphocyte and plasma cell infiltrate. ${ }^{8}$ These findings are not sufficient, however, and immunohistochemistry is necessary for definite diagnosis. Nearly $100.0 \%$ of cases are strongly positive S100 protein, CD56 and vimentin in nearly $100.0 \%$ of cases. Glial fibrillary acidic protein is found in about $75.0 \%$ of shwannomas, including with negative CD117 (c-kit) or DOG-1 (detail in GISTs) and negative for smooth muscle actin (SMA) or desmin (detail in leiomyoma) is definite diagnosis of gastric schwannoma (Figure 4). ${ }^{6,8}$

Table 4 Endoscopic ultrasound (EUS) findings in subepithelial tumors ${ }^{15}$

\begin{tabular}{|c|c|c|c|}
\hline Tumor & EUS layer & Organ & EUS appearance \\
\hline Schwannoma & $4^{\text {th }}\left(3^{\text {rd }}\right)$ & Stomach & Hypoechoic, round or oval, well demarcated. \\
\hline Gastrointestinal stromal tumors & $4^{\text {th }}\left(2^{\text {nd }}, 3^{\text {rd }}\right)$ & Stomach & $\begin{array}{l}\text { Hypoechoic, round or oval, well demarcated. (large tumor }>4 \mathrm{~cm} \text {, } \\
\text { homogeneous, irregular border, cystic areas of echogenic foci) }\end{array}$ \\
\hline Leiomyoma & $4^{\text {th }}\left(2^{\text {nd }}\right)$ & Stomach & Hypoechoic, round or oval, well demarcated. \\
\hline Leiomyosarcoma & $4^{\text {th }}\left(2^{\text {nd }}\right)$ & Stomach & $\begin{array}{l}\text { Hypoechoic, heterogeneous, irregular extraluminal border or invasive } \\
\text { to adjacent organs. }\end{array}$ \\
\hline
\end{tabular}

Table 5 Endoscopic ultrasound findings in gastric schwannoma and low risk and high risk gastrointestinal stromal tumors (GISTs) ${ }^{16}$

\begin{tabular}{llll}
\hline Appearance & Schwannoma & Low-risk GISTs & High-risk GISTs \\
\hline Echogenicity & $\begin{array}{c}\text { Heterogeneous and hypoechoic, } \\
\text { but slightly higher than MP }\end{array}$ & Homogeneous and hypoechoic & Heterogeneous and hypoechoic \\
Halo & Frequent & Uncertain & Uncertain \\
Growth & In<out (mostly) & In>out (mostly) & Variety \\
Margin & Regular & Regular & Irregular \\
Lobulation & Rare & Uncommon & Common \\
High echo spot & Common & Occasional & Common \\
Cyst & Very rare & Frequent & Very frequent \\
Calcification & Scarce & Occasional & Occasional
\end{tabular}



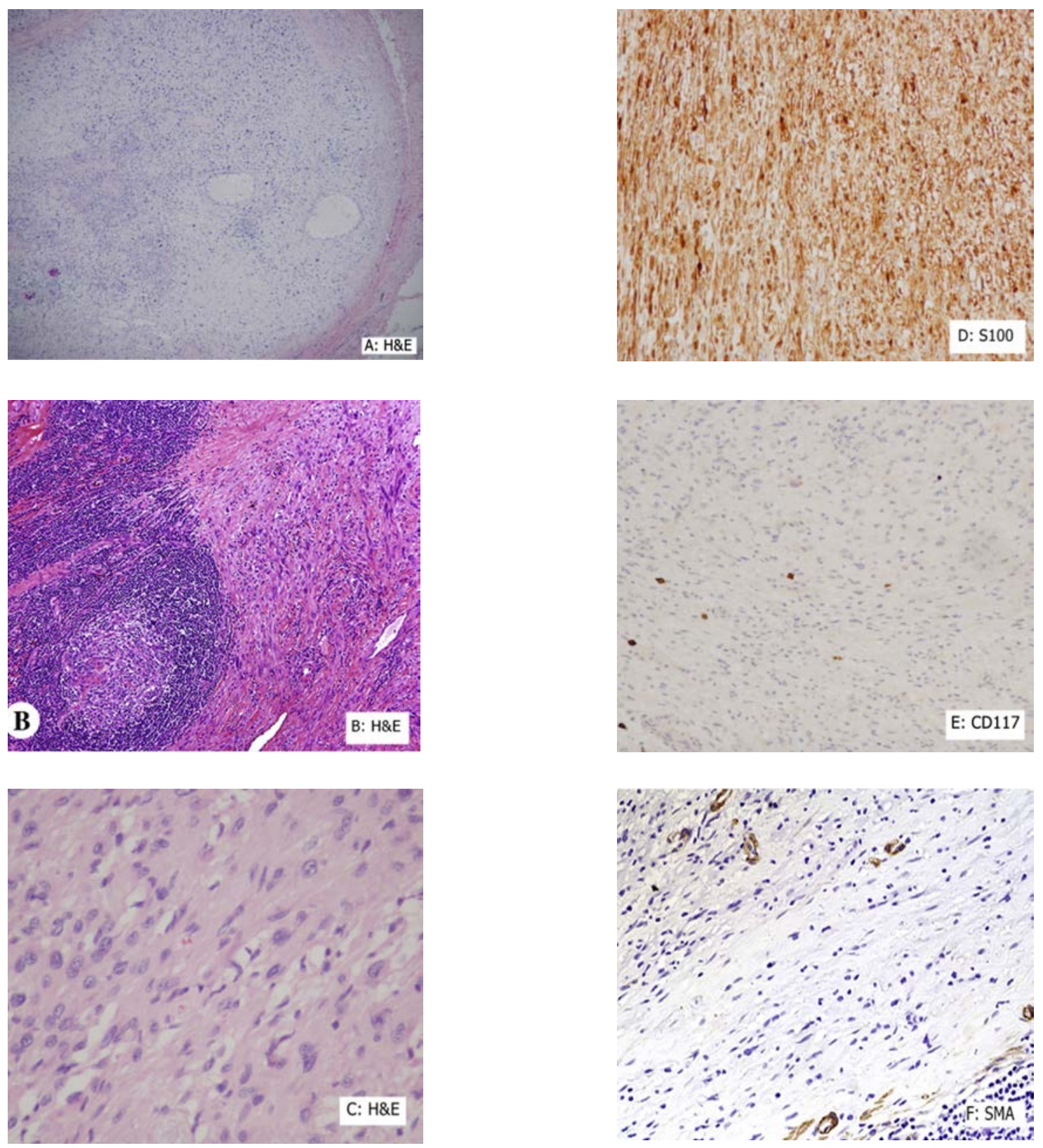

Figure 4 Microscopic and immunohistochemistry findings of gastric schwannoma
A, B, C: tumor with thin capsules with peripheral lymphoid cuff with occasional germinal center, with spindle cell and nuclear palisade appearance.
D: marked positive S100 protein (S100 is a specific marker to schwannoma)
E: negative CD117 (CD117 is a specific marker to GISTs)
F: negative SMA (SMA is a specific marker to leiomyoma) 
Microscopic examination of a gastric schwannoma is necessary for evaluation of the malignancy of the tumor from nuclear atypia and mitotic count by low, moderate and high $(<5,6-10,>10 \text { per } 50 \text { high-power field, respectively })^{18}$ and a high Ki67 expression index consider to malignant schwannoma. ${ }^{6,8}$

The other common differential diagnosis items of an S100 positive tumor in the stomach are clear cell sarcoma of the stomach and metastatic malignant melanoma of the stomach. ${ }^{8}$

\section{Management}

There are now to date some 200 reported cases of gastric schwannoma, with no specific guideline for management of this tumor, with the endoscopists recommending follow the practical guideline in management subepithelial tumors, based on the symptoms of the patient, tumor size and histologic grading (malignancy potential). ${ }^{17-20}$

1. Symptoms associated with tumor: a tumor with upper Gl bleeding, abdominal pain or gastric outlet obstruction needs surgical or endoscopic removal.

2. Asymptomatic with tumor size more than 5 $\mathrm{cm}$ : consider surgical removal for definite diagnosis and histologic examination for malignancy potential.

3. Asymptomatic with tumor size $2-5 \mathrm{~cm}$ : consider further histologic evaluation by EUS FNA

-GISTs: if the tumor is considered to have malignancy potential, surgical or endoscopic removal should be considered.

-Gastric schwannoma: benign behavior, needs close follow up for 6-12 months, if tumor grows during the follow up period consider surgical or endoscopic removal.

4. Asymptomatic with tumor size less than $2 \mathrm{~cm}$ : close follow up with EUS or CT scan for 6-12 months, if tumor grows during the follow up period consider surgical or endoscopic removal.
Surgical resection is the treatment of choice in gastric schwannoma. Symptomatic patients or large tumor size with malignant features are indication for complete resection. The size and location of the tumor, as well as its relation to the surrounding organs, are important factors in determining the operative technique. Wedge resection, partial, subtotal, or total gastrectomy, or laparoscopy are the normal option. ${ }^{21}$ Endoscopic treatment is a less invasive procedure for tumor resection but the procedure needs an experienced endoscopist. A previous study reported successful tumor resection without serious complications, but future studies are needed to compare techniques and outcomes. $^{22}$

\section{Prognosis}

The gastric schwannoma is a primary benign gastric mesenchymal tumor, rarely malignant, with excellent prognosis after complete removal. Although large tumor size and high mitotic histologic gradingn(>10 per 50 high-power field (HPF)) are not independent factors for confirmation of malignancy. Contrast with GISTs, the larger tumor size with high mitotic histologic grading (>10 per $50 \mathrm{HPF}$ ) also have higher risk to malignancy. ${ }^{6,8,23}$

\section{Conclusion}

The gastric schwannoma is a rare, benign, primary gastric mesenchymal tumor, usually asymptomatic, with about 2-4 times higher incidence in females than males, most commonly occurring during the $5^{\text {th }}-6^{\text {th }}$ decades of life. Cross-sectional imaging (CT or MRI) demonstrate a non-specific finding, difficult to differentiate from other subepithelial tumor. An endoscopic ultrasound can be used to identify the origin of the tumor and its intra-tumoral components, however tissue histology and immunohistochemistry are necessary for confirmation. 


\section{Management of this tumor depends on the} symptoms of the patient, tumor size and mitotic activity as revealed by histology, but most cases have an excellent prognosis without recurrence or meta-stasis after complete surgical or endoscopic removal.

\section{References}

1. Lin CS, Hsu HS, Tsai CH, Li WY, Huang MH. Gastric schwannoma. J Chin Med Assoc 2004;67:583-6.

2. McNeer G, Pack GT. Neoplasms of the stomach. Philadephia: J.B. Lippincott; 1974.

3. Hou YY, Tan YS, Xu JF, Wang XN, Lu SH, Ji Y, et al. Schwannoma of the gastrointestinal tract: a clinicopathological, immunohistochemical and ultrastructural study of 33 cases. Histopathology 2006;48:536-45.

4. Daimaru $Y$, Kido $H$, Hashimoto H, Enjoji M. Benign schwannoma of the gastrointestinal tract: a clinicopathologic and immunohistochemical study. Humpathol 1988;19:257-64.

5. Miettinen M, Majidi M, Lasota J. Pathology and diagnostic criteria of gastrointestinal tumors (GISTs): a review. Eur J Cancer 2002;38:39-51.

6. Miettinen M, Sobin LH, Lasota J. Gastrointestinal stromal tumors of the stomach: a clinicopathologic, immunohistochemical, and molecular genetic study of 1,765 cases with long-term follow-up. Am J Surg Pathol 2005;29:52-68.

7. Diaz ST, Hansson CM, de Bustos C, Mantripagada KK, Piotrowski A, Benetkiewicz M, et al. High-resolution array-CGH profiling of germline and tumor-specific copy number alterations on chromosome 22 in patients affected with schwannomas. Hum Genet 2005;118:35-44.

8. Lysandra V, Rebecca M, Jerzy L, Miettinen M. Gastric schwannoma: a clinicopathologic study of 51 cases and critical review of the literature. Hum Pathol 2012;43:650-9.

9. Evans DG. Neurofibromatosis type 2 (NF2): a clinical and molecular review. Orphaned J Rare Dis 2009;4:16.

10. Agaimy A, Markl B, Kitz J, Wunsch PH, Arnholdt H, Fuzesi L, et al. Peripheral nerve sheath tumors of the gastrointestinal tract: a multicenter study of 58 patients including NF1-associated gastric schwannoma and unusual morphological variants. Virchows Arch 2010;456:411-22.

11. Agaimy A, Markl B, Kitz J. Peripheral nerve sheath tumors of the gastrointestinal tract: a multicenter study of 58 patients including NF1-assocated gastric schwannoma and unusual morphologic variants. Virchows Arch 2010;456:411-22.

12. Hong HS, Ha HK, Won HJ, Byun JH, Shin YM, Kim AY, et al. Gastric schwannoma: radiological features with endoscopic and pathological correlation. Clin Radiol 2008;63:536-42.

13. Takeda M, Amano Y, Machida T, Kato S, Naito Z, Kumita S. CT, MRI, and PET findings of gastric schwannoma. Jpn J Radiol 2012;30:602-5.

14. Yoon W, Paulson K, Mazzara P. Gastric schwannoma: a rare but important differential diagnosis of a gastric submucosal mass. Case Rep Surg 2012;2012:280982.

15. Hiroki S, Kitano M, Kudo M. Diagnosis of subepithelial tumors in the upper gastrointestinal tract by endoscopic ultrasonography. World J Radiol 2010;28:289-97.

16. Zhong D, Wang C, Xu J, Chen MY, Cai JT. Endoscopic ultrasound features of gastric schwannomas with radiological correlation: a case series report. World J Gastroenterol 2012; 18:7397-401.

17. Hwang JH, Rulyak SD, Kimmey MB. American gastroenterological association institute technical review on the management of gastric subepithelial masses. Gastroenterology 2006; 130:2217-28.

18. Mekky MA, Yamao K, Sawaki A, Mizuno N, Hara K, Nafeh MA, et al. Diagnosis utility of EUS-guided FNA in patients with gastric submucosal tumors. Gastrointest Endosc 2010;71: 913-9.

19. Sepe PS, Brugge WR. A guide for the diagnosis and management of gastrointestinal stromal cell tumors. Nat Rev Gastroenterol Hepatol 2009;6:363-71.

20. Kongkam P, Devereaux BM, Ponnudurai P, Rattanachu-ek T, Sahai AV, Gotoda T, et al. Endoscopic ultrasound forum summary from the Asian Pacific digestive week. Endosc Ultrasound 2013;2:43-60.

21. Bandoh $T$, Isoyama $T$, Toyoshima $H$. Submucosal tumors of the stomach: a study of 100 operative cases. Surgery 1993; 113:498-506.

22. Cai MY, Xu JX, Zhou PH, XU MD, Chen SY, Hou J. Endoscopic resection for gastric schwannoma with long-term outcomes. Surg Endosc 2016;30:3994-4000.

23. Atmatzidis $S$, Chatzimavroudis $G$, Dragoumis $D$, Tsiaousis $P$, Patsas A, Atmatzidis K. Gastric schwannoma: a case report and literature review. Hipokratia 2012;16:280-2. 